Quantum effects

\title{
Heat flow in atomic bottlenecks
}

The Wieldmann-Franz law linking electrical and thermal conductance has now been experimentally verified in atomic junctions.

\section{Oleg Kolosov}

For more than a decade now, the excess heat generated by the flow of electrical currents has stalled the speed of modern microprocessors [1]. This, and other phenomena in which heat and electricity are close partners are difficult to study at the nanoscale and are extremely challenging as features approach the atomic dimensions. Writing in Nature Nanotechnology, Mosso et al. now present measurements of heat conductivity and electrical current in gold junctions only a few atoms across [2]. The authors experimentally confirm that the smallest amount of heat that can be carried across the metallic junction -- a single quantum of heat - is directly proportional to the quantum of electrical conductance through the same junction. Although quantization of electrical conductance in junctions was predicted by Rolf Landauer in 1957 [3] and the experimental observation of quantization of the heat flow reported in $\mathbf{2 0 0 0}[4,5]$, the definitive link between these two very different quantum phenomena at the nanoscale was yet to be confirmed.

Thermal energy in solids is stored in the collective vibrations of atoms - called phonons. They can carry heat from hot to cold regions. Whereas in insulators and semiconductors, heat transfer is dominated by phonons, in metals the majority of heat is carried by free electrons. Free electrons can move at high speed and are also responsible for the good electrical conduction observed in metals. In bulk metals, the link between movement of heat and electrical current was empirically established by Gustav Wiedemann and Rudolph Franz in 1853, as they showed that electrical and thermal conductivities scale with each other. This link is much less certain as the size of the wires approaches nanometre and atomic length scales, the dimensions that are of ultimate interest for modern electronics and sensors.

In order to establish, or disprove the link between electrical and thermal conductivity at atomic scale, Mosso et al. had to overcome the experimental challenge of creating an atomically thin junction, thus creating a bottleneck for both electrical current and heat flow. They then had to measure these two variables simultaneously at varying diameters of the junction. To do so, they built a special microelectromechanical (MEMS) device consisting of a thin silicon nitride membrane of few hundreds micrometres across with a platinum resistive heater that served as a thermometer (it can sense changes of its electrical resistance with temperature). Next to the heater they placed a gold landing pad that can be approached by a finely etched gold tip mounted on a scanning tunnelling microscope (STM) scanner. Approaching and retracting the STM tip to and from the gold pad created a nanoscale junction with varying dimensions. In order to make MEMS detector highly sensitive to vanishingly small heat flows in the junction, a detector was suspended at four $200-\mu \mathrm{m}$ long and 100-nm thick tethers. The setup was placed in vacuum, with all experiments performed in a noise free laboratory. While to make and break atomic junctions in STM is a relatively straightforward feat [6], the novel feature of the setup is the detector. The new MEMS provided needed thermal sensitivity, but unfortunately it was too flexible for out-of-plane movements with minute attractive forces of the approaching STM tip displacing MEMS like a trampoline. As this does 
not allow atomic scale control of the distance between the gold platform and the tip, the team solved this problem by using the high in-plane stiffness of the tethered platform, and glancing angle of landing and removal of the STM tip from the surface. By doing so, Mosso et al. were able to control the tip side contact with the gold pad through the presence of atomic scale roughness. The result was not short of spectacular - the tip-surface contact with initial size of several tens of atoms across, became thinner as the STM tip retracted down to a single atom. The electrical and thermal conductance then revealed a synchronous step-wise decrease indicative of quantization of both heat and electrical current transfer, with a final step, before breaking contact, corresponding to a single quantum of thermal and electrical conductivity (see figure).

This correlation confirms that the Wiedemann-Franz law can now be safely used to predict and to explore nanoscale thermal and electrical phenomena down to the size of few atoms or a single molecule [7]. Although these results are applicable only to metallic junctions, where electrons govern the conductance of both heat and electrical current, Mosso et al.'s work will stimulate research in metal-semiconductor and semiconductor-semiconductor junctions as in where phonons play a major role in heat transport.

Oleg Kolosov is at the Department of Physics, Lancaster University, Lancaster, UK.

\section{References}

1. Pop, E.,Energy Dissipation and Transport in Nanoscale Devices. Nano Res. 2010, 3, 147-169.

2. Mosso N., et al. Nature Nanotechnology XX, XX, XXX

3. Lee, P. A.; Ramakrishnan, T. V. Reviews of Modern Physics 1985, 57, (2), 287-337

4. Pendry, J. B. Quantum limits to the flow of information and entropy. J. Phys. A 16, 2161-2171 (1983)

5. Schwab, K., et al. Nature 2000, 404, (6781), 974-977]

6. Pascual, J. I., et al. Physical Review Letters 1993, 71, (12), 1852-1855

7. Evangeli, C., et al. Nano Letters 2015, 15, (2), 1006-1011. 


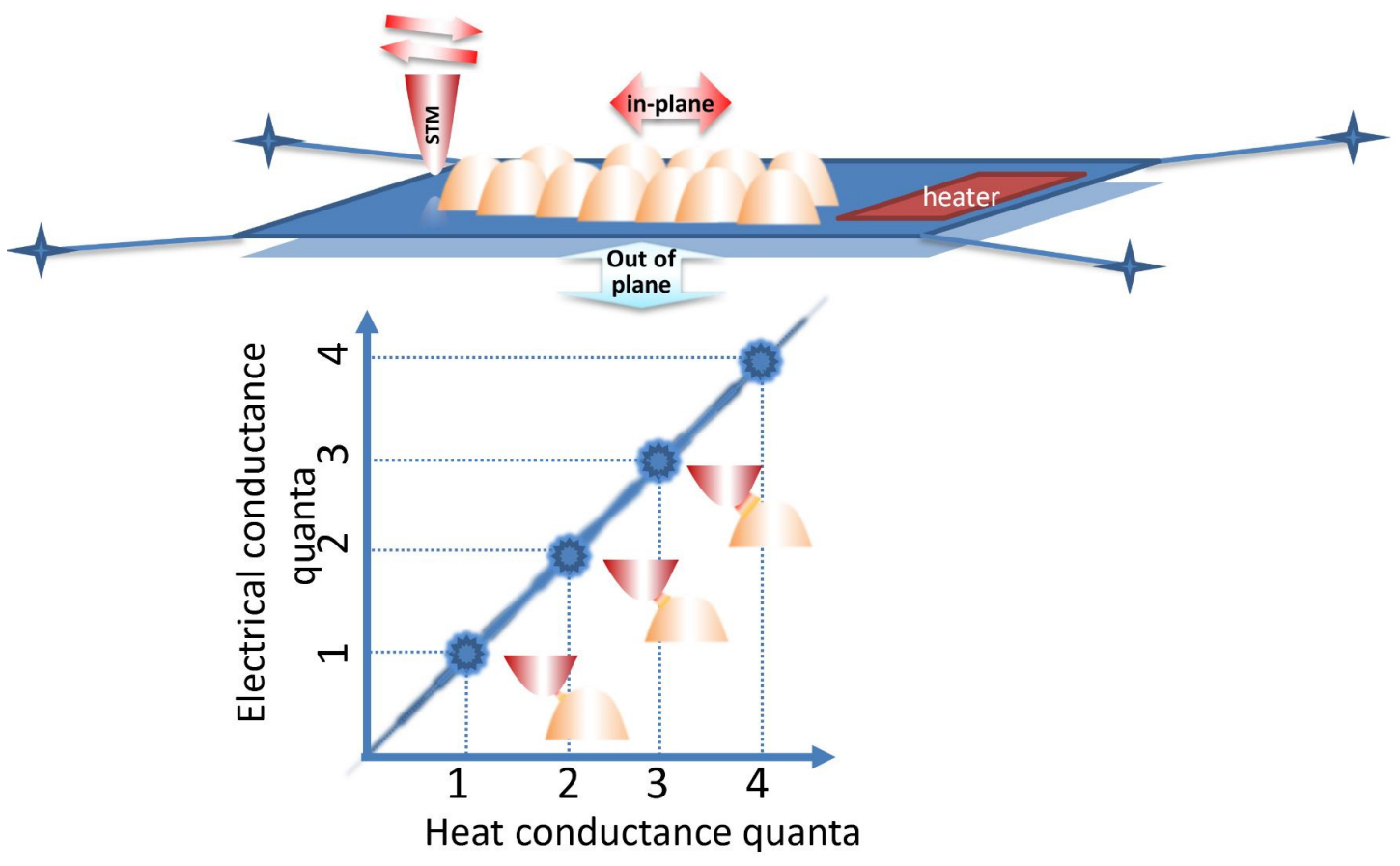

Figure 1. Comparing single quanta of heat and electrical conductance requires both creation of few atoms wide junctions between two metals as well as detection of vanishingly small amounts of heat flow. Mosso et al. achieved this in a special Micro-Electro-Mechanical Sensing (MEMS) device with the integrated heater and temperature sensor that was maximally thermally isolated from the supporting chip by a long low thermal conductance tethers. In their setup a sharpened scanning tunnelling microscope (STM) Au tip approaches an Au coated area of the MEMS with the heat and current flowing to the tip measured by the sensitive electronics. The MEMS that behaved like a micro-trampoline, turned out too flexible for the out-of plane movement, preventing precise control of the atomic junctions. To overcome this, a team used a glancing angle of landing and retracting of the STM tip on the Au platform, with high in-plane stiffness of stretched tethers allowing to create stable atomic-size junctions. As the junction size varied, measurements of heat and electrical conductance showed a simultaneous spikes in both conductances corresponding to individual quanta of heat and electrical conductance. This supported a Wiedemann-Franz law established centuries ago hat links electrical and thermal conductance, now down to the atomic level and to the individual conductance quanta of heat and electrical current. 
